\title{
Microscopy
}

\section{Optimisation of digital volume correlation computation in SR-microCT images of trabecular bone and bone-biomaterial systems}

\begin{tabular}{|r|l|}
\hline Journal: & Journal of Microscopy \\
\hline Manuscript ID & Draft \\
\hline Wiley - Manuscript type: & Original Article \\
\hline Date Submitted by the Author: & n/a \\
\hline Complete List of Authors: & $\begin{array}{l}\text { Peña Fernández, Marta; University of Portsmouth, School of Engineering } \\
\text { Barber, Asa; University of Portsmouth, School of Engineering; London } \\
\text { South Bank University, School of Engineering } \\
\text { Blunn, Gordon; University of Portsmouth, School of Pharmacy and } \\
\text { Biomedical Sciences } \\
\text { Tozzi, Gianluca; University of Portsmouth, School of Engineering }\end{array}$ \\
\hline Keywords: & $\begin{array}{l}\text { digital volume correlation, synchrotron, microCT, bone, bone-biomaterial, } \\
\text { displacement/strain uncertainties }\end{array}$ \\
\hline & \\
\hline
\end{tabular}


Page 1 of 32

Journal of Microscopy

OPTIMISATION OF DIGITAL VOLUME CORRELATION COMPUTATION IN SRMICROCT IMAGES OF TRABECULAR BONE AND BONE-BIOMATERIAL SYSTEMS

Marta Peña-Fernández ${ }^{1 *}$, Asa H. Barber ${ }^{1,2}$, Gordon Blunt ${ }^{3}$, Gianluca Tozzi ${ }^{1}$

Affiliations:

1. School of Engineering, University of Portsmouth, Portsmouth, UK.

2. School of Engineering, London South Bank University, UK.

3. School of Pharmacy and Biomedical Sciences, University of Portsmouth, Portsmouth, UK.

*Corresponding author:

Marta Peña Fernández

School of Engineering

University of Portsmouth

Anglesea Building, Anglesea Road

P01 3DJ, Portsmouth

United Kingdom

Tel: +44 (0)239284 2543

Email: marta.pena-fernandez@port.ac.uk

1 


\begin{abstract}
A micromechanical characterization of biomaterials for bone tissue engineering is essential to understand the quality of the newly regenerated bone, enabling the improvement of tissue regeneration strategies. A combination of micro-computed tomography (microCT) in conjunction with in situ mechanical testing and digital volume correlation (DVC) has become a powerful technique to investigate the internal deformation of bone structure at a range of dimensional scales. However, in order to obtain accurate three-dimensional (3D) strain measurement at tissue level, high-resolution images must be acquired, and displacement/strain measurement uncertainties evaluated. The aim of this study was to optimize imaging parameters, image post-processing and DVC settings to enhance computation based on 'zerostrain' repeated high-resolution synchrotron microCT (SR-microCT) scans of trabecular bone and bone-biomaterial systems. Low exposures to SR X-ray radiation were required to minimize irradiation-induced tissue damage, resulting in the need of advanced $3 D$ filters on the reconstructed images to reduce DVC-measured strain errors. Furthermore, the computation of strain values only in the mineralised material (i.e. bone, biomaterial) allowed the exclusion of large artifacts localised in the bone marrow. This study demonstrated the suitability of a local DVC approach based on SR-microCT images to investigate the micromechanics of trabecular bone and bonebiomaterial composites at tissue level with a standard deviation of the errors $\sim 100$ microstrain after a thorough optimization of DVC computation.
\end{abstract}

Keywords: digital volume correlation, synchrotron, microCT, bone, bone-biomaterial, displacement/strain uncertainties 


\section{INTRODUCTION}

Novel osteoregenerative biomaterials for bone tissue engineering are constantly under development with the aim of favouring optimal bone integration in the defect site up to complete bone formation (Wang \& Yeung 2017; Tozzi et al., 2016; Stevens 2008). Synthetic bone grafts substitutes, such as commercial StronBone (Sriranganathan et al., 2016), have shown excellent regenerative properties (Dorozhkin, 2013; García-Gareta et al., 2015; Wang \& Yeung, 2017). However, to date, the ability of such biomaterials in producing bone that is comparable to the native tissue they are meant to replace is poorly understood and may, therefore, be insufficient to support load-bearing regions. Micromechanical characterization of bone-biomaterial systems has been extremely beneficial to better understand the overall structure response of such composites (Tozzi et al., 2012, 2014, 2016; Danesi et al., 2016). Particularly, a significant understanding of the internal microdamage at the bone-biomaterial interface, which could promote further damage to the bone structure, remains partially unexplored (Tozzi et al., 2016; Danesi et al., 2016). This is due to the intrinsic limitations of most experimental techniques, like strain gauges or digital image correlation, limited to two-dimensional surface measurement, while the internal volume response could not be interrogated (Palanca et al., 2015; Grassi \& Isaksson, 2015).

The recent advances in high-resolution micro-computed tomography (microCT) combined with in situ mechanical testing (Buffiere et al., 2010; Nazarian \& Müller, 2004), has allowed Digital Volume Correlation (DVC) to become a powerful and unique technique to investigate three-dimensional (3D) full-field displacement and strain in bone based on 3D images acquired at different deformation states (Bay et al., 1999; Grassi \& Isaksson, 2015; Roberts et al., 2014). DVC has been extensively used to investigate trabecular bone (Gillard et al., 2014; Liu \& Morgan, 2007), cortical bone (Christen et al., 2012), whole bones (Tozzi et al., 2016; Hussein et al., 2012;), biomaterials (Madi et al., 2013a), and bone-biomaterial systems (Danesi et al., 2016; Tozzi et al., 2012) under different loading conditions. However, in order to expand the applications of DVC to the study of clinically-relevant issues such as the integration of biomaterials after bone grafting procedures, it is important to understand the error associated with the DVC measurement and optimising the imaging and DVC settings to minimise these errors. In this perspective, strain uncertainties of any specific DVC approach are usually quantified on repeated scans (i.e. in a known deformation field such as 'zero-strain') to account for the intrinsic noise of the input images (Dall'Ara et al., 2017; Dall'Ara et al., 2014). This repeated scan methodology has been already adopted to quantify strain errors associated with bone-biomaterial interfaces (i.e. bone-cement) (Tozzi et al., 2017; Palanca et al., 2016). Specifically, Tozzi et al. (2017) focused on images obtained in laboratory microCT systems with a voxel size of $39 \mu \mathrm{m}$. The random errors for the strain components were found to be all around or lower than $200 \mu \varepsilon$, for a sub-volume size of 48 voxels, providing measurements approximately every $2 \mathrm{~mm}$. In this sense, the 
DVC measurement spatial resolution were able to include more bone structural units (BSUs, i.e., trabecula), and enabled the classification of regions at high or low localised strain. However, measurement within BSUs remained unexplored. To overcome the limitation of laboratory microCT systems, where a strong compromise between strain precision and measurement spatial resolution must be accepted (Palanca et al., 2015; Dall'Ara et al., 2014), synchrotron-based microCT (SRmicroCT) has proven to provide strain uncertainties below $200 \mu \varepsilon$ for correlations performed with a measurement spatial resolution below $100 \mu \mathrm{m}$ for both cortical and trabecular bone (Palanca et al., 2017). In fact, the use of DVC based on SR-microCT high resolution images ( 2 $\mu \mathrm{m}$ voxel size), allowed reliable strain measurements within the BSUs (Dall'Ara et al., 2017; Palanca et al., 2017). However, the performance of DVC based on SR-microCT images on composite biological structures such as bone-biomaterial systems remained unclear. Recently, Dall'Ara et al. (2017) provided an overview of the strain errors associated to several bone structures acquired with different microCT techniques, at different dimensional levels. In that study, the precision of DVC applied to SR-microCT images of bone and bone-biomaterial systems was analysed for the first time, reporting values below $150 \mu \varepsilon$ for a measurement spatial resolution close to $150 \mu \mathrm{m}$, allowing strain measurement at tissue level. Despite those results were promising to evaluate fullfield strain in bone-biomaterial systems, only the precision in terms of displacement and strain was analysed. In addition, the optimisation on imaging settings, postprocessing and DVC features was not reported.

The reliability of DVC based on high-resolution SR-microCT images of trabecular bone and bone-biomaterial systems will provide accurate 3D strain measurement at tissue level. Therefore, a better understanding of the micromechanical behaviour of bone-biomaterials interfaces can be achieved through optimisation of the DVC computation. In this perspective, the main aim of this study was to investigate the effect of image post-processing and DVC settings on the displacement- and strainmeasured uncertainties at tissue level, using a local DVC approach based on 'zerostrain' repeated SR-microCT scans, in trabecular bone and bone-biomaterial systems.

\section{METHODS}

\section{Specimen preparation}

Cylindrical bone defects (8mm diameter by $14 \mathrm{~mm}$ depth) were surgically created in the femur condyles of an adult sheep (Coathup et al., 2016) and four different biomaterials (Actifuse, ApaPore, StronBone, StronBone-P) were implanted under Ethics approval granted by the Royal Veterinary College and in compliance with the United Kingdom Home Office regulations (Animal Scientific Procedure Act [1986]). Six weeks after implantation both left and right condyles were harvested and cylindrical samples ( $4 \mathrm{~mm}$ diameter by $18 \mathrm{~mm}$ length) were cored from the condyles in proximal-distal direction by drilling with a coring tool. The ends of the cores were 
trimmed plane and parallel, and end-constraint was achieved by embedding the ends of the samples in poly-methyl-methacrylate (PMMA) endcaps. Approximately, 5 $\mathrm{mm}$ of the core was embedded into each endcap to achieve a 2:1 aspect ratio and reduce experimental artifacts (Keaveny et al., 1993). In total, four bone-biomaterial systems ( $n=1 \mathrm{p} /$ biomaterial) from the bone defect areas and two trabecular bone controls $\left(n=1\right.$ p/condyle) were analysed. Samples were kept frozen at $-20^{\circ}$ and thawed for around $2 \mathrm{~h}$ in saline solution at room temperature before image acquisition.

\section{SR-microCT}

SR-microCT imaging was performed at the Diamond-Manchester Imaging Branchline 113-2 of Diamond Light Source (DLS), UK, using a filtered $(1.3 \mathrm{~mm}$ pyrolytic graphite, $3.2 \mathrm{~mm}$ aluminium and $60 \mu \mathrm{m}$ steel) partially-coherent polychromatic 'pink' beam (5-35 keV) of parallel geometry with an undulator gap of 5 $\mathrm{mm}$. Sample alignment in the beam was under low dose conditions (undulator gap of $10 \mathrm{~mm}$ ). Projections were recorded by a sCMOS (2560 x 2160 pixels) pco.edge 5.5 (PCO AG, Germany) detector, which was coupled to a $500 \mu \mathrm{m}$-thick $\mathrm{CdWO}_{4}$ and a visual light microscope. A $1.25 \mathrm{X}$ objective lens was used to achieve a total magnification of 2.5X, resulting in an effective voxel size of $2.6 \mu \mathrm{m}$ and a field of view of $6.7 \times 5.6 \mathrm{~mm}$. For each dataset, 1801 projection images were collected over 180 degrees of continuous rotation ('fly scan'). The final projection was not used for reconstruction, but was compared to the first image to check for experimental problems including sample deformation and bulk movements (Atwood et al., 2015). The exposure time was set to $64 \mathrm{~ms}$ per projection in order to minimize SR irradiation-induced damage during image acquisition (Peña-Fernández et al., 2018) (Fig. 1). The propagation distance (sample to detector) was increased in $\sim 100 \mathrm{~mm}$ increments until sufficient in-line phase contrast was gained to visualise the microstructure. The final propagation distance used was $150 \mathrm{~mm}$. The projection images were flat and dark corrected prior to reconstruction. For each dataset, 40 flat and dark images were collected. Reconstruction was performed at DLS using the inhouse software, DAWN (Basham et al., 2015; Titarenko et al., 2010), incorporating ring artifact suppression. Specimens were imaged within a loading stage (CT5000, Deben Ltd, UK) equipped with an environmental chamber and were kept immersed in saline solution during the entire experiment. A small preload $(5 \mathrm{~N})$ was applied only to ensure good end contact prior to imaging and minimise motion artifacts during the scan. Each specimen was scanned twice under the same configuration ('zero-strain' repeated scan) without any repositioning.

\section{Image post-processing}

Each 3D image dataset consisted of 2160 images $(2256 \times 2076$ pixels) with 32-bit graylevels. Images were converted to 8 -bit grayscale $(0-255$ counts). The repeated scans for each specimen were first rigidly registered using Fiji software (Schindelin et al., 2012). Registration was performed minimising the Euclidean difference 
between the reference and the target image, followed by a resampling using a cubic spline interpolation (Meijering et al., 2001). After registration, a volume of interest (VOI) was cropped for each tomogram, consisting of a parallelepiped with side lengths of 1000 voxels $\left(2.6 \mathrm{\mu m}^{3}\right)$. The VOI was set in the centre of the volume for the controls and manually selected for the bone-biomaterial specimens in order to include the interface. Noise in the images was reduced by applying a 3D filter. To compare the DVC algorithm performance on the filtered images, two different filters were used (Fig. 2): a median filter (radius $=2$ pixels) and a non-local means (NLM) filter (Buades et al., 2011; Darbon et al., 2008), where the variance (sigma) of the noise was automatically estimated for each dataset (Immerkær 1996).

Additionally, the original SR-microCT images were also masked by setting to zero the grayscale intensity of non-bone/biomaterial voxels. A binary image (value one for bone-biomaterial voxels and zero elsewhere) was first created form the non-local mean denoised images using an iterative approach. Due to the low exposure used during image acquisition to minimise irradiation-induced damage in the tissue, the quality of the images was considerably low (Fig. 1); therefore, a global thresholding can be insufficient for segmenting the mineralised tissue and biomaterial from the soft (i.e. bone marrow) and watery material, due to variations in signal intensity and noise within the same material. The method employed in this study firstly used a global threshold based on Huang's method (Huang \& Wang, 1955), followed by an iterative approach. The latter consisted on applying three different operations to the binary images as follows: 1) the connected regions in the 3D volume were identified by applying a purifying cycle using BoneJ (Doube et al., 2010), which locates all particles in the 3D volume and removes all of them but the largest foreground (bonebiomaterial) and background (bone marrow) particles (Odgaard \& Gundersen, 1993); 2) a closing cycle, which performs a dilation operation followed by erosion, was applied in order to fill in small holes; 3) an opening cycle, consisting on an erosion operation followed by dilation, was used to remove isolated pixels. The quality of the binary images was checked by visual inspection after each iteration. The iterative process was concluded when no improvements were observed. In this study 6 iterations were selected (Fig. 3). Masked images, with the original greyscale value in the mineralised tissue and biomaterial, and zero elsewhere, were obtained multiplying the filtered to the final binary image. For each VOI, the solid volume fraction (SV/TV) was obtained to assess possible correlations with DVC measurements.

\section{Digital volume correlation}

DVC was performed on the reconstructed tomograms using DaVis 8.4 software (LaVision Ltd., Goettingen, Germany). DaVis is a cross-correlation method operating on the intensity values (graylevel) of 3D images. In essence, the measurement volume is divided into smaller sub-volumes and the contrast pattern within the subvolumes is then tracked from reference to deformed state, independently (local 
approach (Madi et al., 2013b)) as a discrete function of the graylevels. The matching between the sub-volumes is achieved via a direct cross-correlation (DC) function (Cheminet et al., 2014). It is called "direct" because it directly sums the products of voxel gray values of the initial and deformed volumes to form the 3D correlation map. The normalised correlation coefficient $C_{\text {norm }}$ for two volumes $A$ and $B$ with shifts $d x, d y, d z$ and a window of $N x N x N$ voxels at point $\left(x_{0}, y_{0}, z_{0}\right)$ is computed according to:

$$
C_{n o r m}(d x, d y, d z)=\sum_{(i, j, k)} \frac{\left(A_{i, j, k}-\langle A\rangle\right)\left(B_{i+d x, j+d y, k+d z}-\left\langle B_{d x, d y, d z}\right\rangle\right)}{\sqrt{\left|A^{\prime}\right|^{2} \cdot\left|B_{d x, d y, d z}^{\prime}\right|^{2}}}
$$

With

$$
\begin{aligned}
& \langle A\rangle=\sum_{(i, j, k)} \frac{A_{i, j, k}}{N^{3}} \\
& \left\langle B_{d x, d y, d z}\right\rangle=\sum_{(i, j, k)} \frac{B_{i+d x, j+d y, k+d z}}{N^{3}} \\
& \left|A^{\prime}\right|^{2}=\sum_{(i, j, k)}\left[A_{i, j, k}-\langle A\rangle\right]^{2} \\
& \left|B^{\prime} d x, d y, d z\right|^{2}=\sum_{(i, j, k)}\left[B_{i+d x, j+d y, k+d z}-\left\langle B_{d x, d y, d z}\right\rangle\right]^{2}
\end{aligned}
$$

Where all summations run from $(i, j, k)=\left(x_{0}, y_{0}, z_{0}\right)$ to $(i, j, k)=\left(x_{0}+N-1, y_{0}+\right.$ $\left.N-1, z_{0}+N-1\right) . A_{i, j, k}$ is the gray value (intensity) of the voxel $(i, j, k)$ in the reference volume $A$, and consequently $B_{i+d x, j+d y, k+d z}$ is the intensity of the voxel at the shifted position $(i+d x, j+d y, k+d z)$ in the deformed volume $B$.

The resulting displacement estimate is obtained by maximising the normalised correlation function for each sub-volume, where sub-pixel accuracy is achieved by fitting a Gaussian curve to the correlation peak (Scarano, 2013). DaVis adopts a multi-pass scheme that uses the displacement gradient from the previous pass to deform the sub-volume on the subsequent pass until the highest possible correlation is achieved (Madi et al., 2013a). Tri-linear interpolation is used to calculate displacements of voxels located between nodes. The final displacement is therefore a 3D full-field average displacement of the pattern within that sub-volume between reference and deformed volume. From the field of resultant displacement vectors at the centre of each sub-volume, the field of strain components is computed using a centered finite difference (CFD) scheme (Germaneau et al., 2007a, 2007b). 


\section{Influence of sub-volume size}

The DVC technique relies on the internal graylevel texture of the material that can be recognised in the 3D images to correlate a reference sub-volume to a deformed one. The features included within each sub-volume, and therefore the sub-volume size affects DVC uncertainties (Liu \& Morgan, 2007; Roberts et al., 2014). A small subvolume size is typically susceptible to noise effects, whereas large sub-volumes may result in an insufficient spatial resolution (Dall'Ara et al., 2014). Therefore, the goal is always to choose a sub-volume small enough to capture the essential features of deformation, and still large enough to give accurate results. In order to evaluate the influence of sub-volume size on the DVC displacement/strain uncertainties, seven sub-volume sizes ranging from 16 to 112 voxels, in steps of 16 voxels were investigated. Two passes were performed for each sub-volume size, using the first pass as a predictor for the final computation and therefore, improving the correlation. Moreover, a multi-pass scheme with variable sub-volume sizes was tested. The multi-pass scheme used sub-volumes of $112,96,80,64$ and 48 voxels, with $0 \%$ overlap between the sub-volumes.

\section{Influence of masking}

DVC was applied to the raw and masked images to investigate the influence of including the bone marrow regions, usually associated to large strain artefacts due to the lack of a clear pattern distribution, in the correlation algorithm. Two different approaches were considered for DVC computation in the mask images: treating the non-mineralised areas as a black 'zero-count' region (mask image) and creating a threshold-based algorithm mask using DaVis tools (DaVis-mask image). The difference between both approaches lies on the inclusion of regions outside the bone-biomaterial when correlating the 3D images. Whereas the use of masked images allows DVC algorithm to correlate the entire 3D image (bone-biomaterial and black regions), DaVis-mask allows calculating vectors only in mineralised (bonebiomaterial) areas within the 3D image. Additionally, the minimal fraction of valid pixel (mfvp) can be controlled when using the masking tools in DaVis. This parameter specifies the number of voxels that need to be contained within a subvolume for the computed vector to be valid; the higher this value the less close calculated vectors are to the mask edges, resulting in a significant loss of data. It should be highlighted that the resulting vectors are computed in the centre of each sub-volume and not at the centre of mass of the non-zero voxels. To account only for the uncertainties within the mineralised material (bone-biomaterial) and allow a better comparison between the three different options, the sub-volumes containing all voxels outside the bone-biomaterial areas were ignored for the raw and masked images, and the uncertainties were computed considering only the remaining subvolumes. The resulting displacement/strain fields were weighted according to the SV/TV for each sub-volume. The number of voxels inside the masked areas. Additionally, for the raw and masked images, sub-volumes with a correlation 
coefficient below 0.6 were removed, to avoid large strain artifacts due to poor correlation. The latter could not be applied to the DaVis-masked images, as the correlation coefficient was considerably low.

\section{Evaluation of DVC uncertainties}

To quantify the level of uncertainties of the DVC measurements, which is associated to imaging conditions, image post-processing and sub-volume size, different scalar indicators were computed for each pair of 'zero-strain' repeated scans. Ideally, also the displacements could be considered null; however, in the real experiment the actual displacements were affected by the inevitable unknown micro-movements of the different parts of the image acquisition setup. Therefore, the systematic error for the displacements could not be quantified and only the random errors, computed as the variability of the displacement within each specimen, was computed (Palanca et al., 2015).

As the test was based on a 'zero-strain' condition, any non-zero values of strain were considered as error. Systematic and random errors for each specimen were calculated as the average and standard deviation for each of the strain components. Additionally, the mean absolute error (MAER) and standard deviation of the error (SDER) were obtained as:

$M A E R=\frac{1}{n} \sum_{k=1}^{n}\left(\frac{1}{6} \sum_{c=1}^{6}\left|\varepsilon_{c, k}\right|\right)$

$S D E R=\sqrt{\frac{1}{n} \sum_{k=1}^{n}\left(\frac{1}{6} \sum_{c=1}^{6}\left|\varepsilon_{c, k}\right|-M A E R\right)^{2}}$

Where $\varepsilon$ represents the strain; $c$ represent the six independent strain components, $k$ represents the measurement point; and $n$ is the total number of measurement points. MAER and SDER correspond to the indicators formerly known as "accuracy" and "precision" (Liu \& Morgan, 2007). Additionally, the correlated volume (CV) was assessed as the volume where correlation was successful. The correlated solid volume (CV/SV) was then computed dividing the CV by the SV/TV.

\section{RESULTS}

\section{Influence of filtering the images.}

The influence of filtering the images was only assessed on the raw images prior to masking procedures and data screening. The use of a median or a NLM filter reduced the noise in the images considerably (Fig. 2) and had a clear impact on the final mean value of the $C_{\text {norm}}$, ranging from 0.41 for the raw images to 0.89 (Table 1 ) 
in the trabecular bone specimens, and from 0.37 to 0.84 in the bone-biomaterial systems, for the raw and NLMD denoised images, respectively, using a multi-pass scheme with a final sub-volume size of 48 voxels. However, the improvement of the correlation coefficient was not related to a clear decrease of the measured DVC errors. Whereas filtering the images slightly reduced the displacement random errors in bone-biomaterial systems (from $0.35 \mu \mathrm{m}$ for the raw images to $0.29 \mu \mathrm{m}$ for the median filtered images and $0.25 \mu \mathrm{m}$ for the NLM filtered images), it slightly increased those values for the trabecular bone specimens (from $0.21 \mu \mathrm{m}$ for the raw images to $0.22 \mu \mathrm{m}$ for the median filtered images and $0.23 \mu \mathrm{m}$ for the NLM filtered images). On the other hand, the use of a NLM filter was found to improve to a small extent the strain uncertainties, whereas the median filter produced slightly higher strain errors, for both trabecular bone specimens and bone-biomaterial systems, when compared to the use of raw images. The same tendencies were observed for the different subvolumes analysed.

Table 1. Mean value of the normalized correlation coefficient $\left(\mathbf{C}_{\text {norm }}\right)$, random errors affecting the displacement components, MAER and SDER on the strain measurements for the multi-pass scheme (final sub-volume size of 48 voxels) in trabecular bone samples and bone-biomaterial systems analysed with the different filters used (raw images, median filter and NLM filter). Median values are calculated accounting for the total number of specimens in each type.

\begin{tabular}{|c|c|c|c|c|c|c|c|}
\hline & \multirow[t]{2}{*}{ Filter } & \multirow[t]{2}{*}{$\mathbf{C}_{\text {norm }}$} & \multicolumn{3}{|c|}{$\begin{array}{l}\text { Displacement random errors } \\
(\mu \mathrm{m})\end{array}$} & \multicolumn{2}{|c|}{ Strain uncertainties $(\mu \varepsilon)$} \\
\hline & & & $x$ & $y$ & Z & MAER & SDER \\
\hline \multirow{3}{*}{$\begin{array}{l}\text { Trabecular } \\
\text { bone }\end{array}$} & None & 0.41 & 0.21 & 0.19 & 0.14 & 387 & 294 \\
\hline & Median & 0.87 & 0.22 & 0.20 & 0.17 & 380 & 273 \\
\hline & NLM & 0.89 & 0.23 & 0.21 & 0.15 & 376 & 257 \\
\hline \multirow{3}{*}{$\begin{array}{l}\text { Bone- } \\
\text { Biomaterial } \\
\text { systems }\end{array}$} & None & 0.37 & 0.35 & 0.22 & 0.14 & 300 & 215 \\
\hline & Median & 0.73 & 0.29 & 0.22 & 0.15 & 307 & 215 \\
\hline & NLM & 0.84 & 0.11 & 0.25 & 0.14 & 289 & 175 \\
\hline
\end{tabular}

Influence of varying the minimal fraction of valid pixels (mfvp).

The mfvp used for DVC computation applied to the DaVis-mask images had an indirect effect on the CV/SV; however, did not modified the $\mathrm{C}_{\text {norm, }}$, displacement random errors or MAER and SDER (Table 2). The CVISV increased from $55.8 \%$ to $86.8 \%$ for the trabecular bone specimens and from $83.0 \%$ to $93.9 \%$ for the bonebiomaterial systems, for a $\mathrm{mfvp}$ of $50 \%$ and $30 \%$, respectively. A visual representation is shown in Fig. 4. The number of successfully correlated sub- 
volumes (colour shaded) increased (higher CV/SV) when decreasing the mfvp. However, the $\mathrm{C}_{\text {norm }}$ remained constant and the slightly changes were found in the displacement random errors and MAER and SDER $(0.07 \mu \mathrm{m}, 69 \mu \varepsilon$, and $66 \mu \varepsilon$, respectively).

Table 2. Correlated solid volume (CV/SV), mean normalized correlation coefficient $\left(\mathbf{C}_{\text {norm }}\right)$, random errors affecting the displacement components, MAER and SDER of the strain measurements for the multi-pass pass scheme (final sub-volume size of 48 voxels) using DaVis-mask images in trabecular bone samples and bone-biomaterial systems varying the minimal fraction of valid pixel ( $\mathbf{m f v p}$ ) for the computation. Median values are calculated accounting for the total number of specimens in each type.

\begin{tabular}{|c|c|c|c|c|c|c|c|c|}
\hline & \multirow[t]{2}{*}{$\begin{array}{c}\text { mfvp } \\
(\%)\end{array}$} & \multirow[t]{2}{*}{$\begin{array}{c}\text { CV/SV } \\
(\%)\end{array}$} & \multirow[t]{2}{*}{$C_{\text {norm }}$} & \multicolumn{3}{|c|}{$\begin{array}{c}\text { Displacement } \\
\text { random errors }(\mu \mathrm{m})\end{array}$} & \multicolumn{2}{|c|}{$\begin{array}{c}\text { Strain } \\
\text { uncertainties }(\mu \varepsilon)\end{array}$} \\
\hline & & & & $x$ & $y$ & $z$ & MAER & SDER \\
\hline \multirow{3}{*}{$\begin{array}{r}\text { Trabecular } \\
\text { bone }\end{array}$} & 50 & 55.8 & 0.41 & 0.42 & 0.50 & 0.34 & 1373 & 736 \\
\hline & 40 & 74.8 & 0.42 & 0.41 & 0.48 & 0.29 & 1308 & 692 \\
\hline & 30 & 86.8 & 0.42 & 0.43 & 0.44 & 0.27 & 1304 & 670 \\
\hline \multirow{3}{*}{$\begin{array}{r}\text { Bone- } \\
\text { Biomaterial } \\
\text { systems }\end{array}$} & 50 & 83.0 & 0.56 & 0.21 & 0.27 & 0.16 & 438 & 337 \\
\hline & 40 & 90.6 & 0.56 & 0.23 & 0.28 & 0.16 & 468 & 395 \\
\hline & 30 & 93.9 & 0.56 & 0.25 & 0.28 & 0.16 & 491 & 384 \\
\hline
\end{tabular}

\section{Influence of masking}

The comparison of masking the images to remove possible artifacts (i.e. bubbles (Fig. 2)) in the marrow/saline solution is presented in Table 3 for the multi-pass scheme with a final sub-volume of 48 voxels. The CV/TV was similar for the three approaches analysed, despite the data was filtered (only sub-volumes with correlation coefficient above 0.6 were considered) for the raw and masked images. The use of DaVis-mask notably decreased the correlation coefficient compared to the use of raw or mask images (from 0.98 to 0.42 in trabecular bone and 0.91 to 0.56 in bone-biomaterial systems). At the same time, the measured uncertainties in terms of strain and displacements were larger. Both mask and raw images showed successful results in terms of correlation coefficient, being slightly higher in the mask case. Furthermore, the MAER and SDER were lower using the masked images. The MAER ranged from $239 \mu \varepsilon$ to $212 \mu \varepsilon$ for the trabecular bone and from $225 \mu \varepsilon$ to 195 $\mu \varepsilon$ for the bone-biomaterial systems in raw and mask images, respectively. Similarly, SDER ranged from $171 \mu \varepsilon$ to $101 \mu \varepsilon$ for trabecular bone and from $107 \mu \varepsilon$ to $102 \mu \varepsilon$ for the bone-biomaterial systems. 
Table 3. Correlated solid volume (CV/SV), normalized correlation coefficient $\left(\mathbf{C}_{\text {norm }}\right)$, random errors affecting the displacement components, MAER and SDER of the strain measurements for the multi-pass pass scheme (final sub-volume size of 48 voxels) using raw, mask and DaVis-mask images in trabecular bone samples and bone-biomaterial systems. Median values are calculated accounting for the total number of specimens in each type.

\begin{tabular}{|c|c|c|c|c|c|c|c|c|}
\hline & \multirow[t]{2}{*}{ Image } & \multirow[t]{2}{*}{$\begin{array}{c}\text { CV/SV } \\
(\%)\end{array}$} & \multirow[t]{2}{*}{$C_{\text {norm }}$} & \multicolumn{3}{|c|}{$\begin{array}{c}\text { Displacement } \\
\text { random errors }(\mu \mathrm{m})\end{array}$} & \multicolumn{2}{|c|}{$\begin{array}{l}\text { Accuracy and } \\
\text { precision }(\mu \varepsilon)\end{array}$} \\
\hline & & & & $x$ & $\mathrm{Y}$ & z & MAER & SDER \\
\hline \multirow{3}{*}{$\begin{array}{r}\text { Trabecular } \\
\text { bone }\end{array}$} & Raw & 92.8 & 0.89 & 0.20 & 0.18 & 0.13 & 239 & 171 \\
\hline & Mask & 86.8 & 0.98 & 0.15 & 0.22 & 0.14 & 212 & 101 \\
\hline & DaVis-mask & 95.5 & 0.42 & 0.43 & 0.44 & 0.27 & 1304 & 670 \\
\hline \multirow{3}{*}{$\begin{array}{r}\text { Bone- } \\
\text { Biomaterial } \\
\text { systems }\end{array}$} & Raw & 94.5 & 0.79 & 0.10 & 0.20 & 0.15 & 225 & 107 \\
\hline & Mask & 90.9 & 0.91 & 0.08 & 0.22 & 0.12 & 195 & 102 \\
\hline & DaVis-mask & 90.0 & 0.56 & 0.25 & 0.28 & 0.16 & 491 & 384 \\
\hline
\end{tabular}

\section{Random errors for the displacement}

The random errors affecting each component of the displacement ranged between $0.42 \mu \mathrm{m}$ and $0.12 \mu \mathrm{m}$ for the raw images, from $0.54 \mu \mathrm{m}$ to $0.14 \mu \mathrm{m}$ for the mask images, and from $0.61 \mu \mathrm{m}$ to $0.13 \mu \varepsilon$ for the DaVis-mask images in the trabecular bone specimens. Similarly, they ranged between $0.40 \mu \mathrm{m}$ and $0.08 \mu \mathrm{m}$ for the raw images, from $0.44 \mu \mathrm{m}$ to $0.11 \mu \mathrm{m}$ for the mask images, and from $0.49 \mu \mathrm{m}$ to $0.08 \mu \varepsilon$ for the DaVis-mask images in the bone-biomaterial systems. Random errors were typically larger for smaller sub-volume sizes (Table 3). The multi-pass scheme (final sub-volume size of 48 voxels) notably improved the performance in both types of specimens for both raw and mask options, when compared to the results obtained with sub-volumes of 48 voxels, obtaining values comparable to the case when a subvolume size of 112 voxels was used. Multi-pass in in DaVis-mask images produced and improvement compared to the single-pass scheme (48 voxels), but less relevant when compared to the raw and mask images. 
Table 4. Random errors of the displacements $(\mu \mathrm{m})$ for the trabecular bone specimens and bone-biomaterial system varying the sub-volume size (voxels) for the raw, mask and DaVis-mask images. Median values are calculated accounting for the total number of specimens in each type.

\begin{tabular}{|c|c|c|c|c|c|c|c|c|c|c|}
\hline & \multirow{2}{*}{$\begin{array}{c}\text { Sub- } \\
\text { volume }\end{array}$} & \multicolumn{3}{|c|}{ Raw } & \multicolumn{3}{|c|}{ Mask } & \multicolumn{3}{|c|}{ DaVis-mask } \\
\hline & & $x$ & $\mathrm{y}$ & $z$ & $x$ & $\mathrm{y}$ & $z$ & $x$ & $\mathrm{y}$ & z \\
\hline \multirow{8}{*}{$\begin{array}{l}\text { Trabecular } \\
\text { bone }\end{array}$} & 16 & 0.35 & 0.42 & 0.29 & 0.38 & 0.54 & 0.35 & 0.61 & 0.61 & 0.26 \\
\hline & 32 & 0.32 & 0.35 & 0.26 & 0.35 & 0.52 & 0.36 & 0.54 & 0.55 & 0.26 \\
\hline & 48 & 0.31 & 0.30 & 0.23 & 0.32 & 0.41 & 0.29 & 0.50 & 0.51 & 0.25 \\
\hline & 64 & 0.26 & 0.31 & 0.20 & 0.28 & 0.36 & 0.26 & 0.53 & 0.52 & 0.36 \\
\hline & 80 & 0.25 & 0.29 & 0.15 & 0.24 & 0.29 & 0.25 & 0.40 & 0.47 & 0.35 \\
\hline & 96 & 0.24 & 0.32 & 0.13 & 0.23 & 0.30 & 0.22 & 0.40 & 0.35 & 0.30 \\
\hline & 112 & 0.20 & 0.20 & 0.12 & 0.21 & 0.25 & 0.19 & 0.21 & 0.28 & 0.13 \\
\hline & $48(\mathrm{mp})$ & 0.20 & 0.18 & 0.13 & 0.15 & 0.22 & 0.14 & 0.43 & 0.44 & 0.27 \\
\hline \multirow{8}{*}{$\begin{array}{l}\text { Bone- } \\
\text { biomaterial } \\
\text { systems }\end{array}$} & 16 & 0.18 & 0.40 & 0.16 & 0.20 & 0.44 & 0.16 & 0.29 & 0.49 & 0.17 \\
\hline & 32 & 0.13 & 0.33 & 0.15 & 0.15 & 0.38 & 0.15 & 0.29 & 0.43 & 0.15 \\
\hline & 48 & 0.12 & 0.28 & 0.13 & 0.12 & 0.36 & 0.14 & 0.24 & 0.37 & 0.14 \\
\hline & 64 & 0.11 & 0.24 & 0.13 & 0.10 & 0.32 & 0.13 & 0.15 & 0.30 & 0.12 \\
\hline & 80 & 0.09 & 0.23 & 0.12 & 0.09 & 0.29 & 0.11 & 0.11 & 0.23 & 0.10 \\
\hline & 96 & 0.08 & 0.21 & 0.12 & 0.08 & 0.23 & 0.12 & 0.10 & 0.20 & 0.09 \\
\hline & 112 & 0.08 & 0.19 & 0.13 & 0.08 & 0.22 & 0.12 & 0.09 & 0.18 & 0.08 \\
\hline & $48(\mathrm{mp})$ & 0.10 & 0.20 & 0.15 & 0.08 & 0.22 & 0.12 & 0.25 & 0.28 & 0.16 \\
\hline
\end{tabular}

\section{MAER and SDER}

To facilitate comparison with publish literature, the scalar values MAER and SDER (Palanca et al., 2016) were computed in order to provide a single strain value associated to each specimen. As expected from the results reported in previous studies (Dall'Ara et al., 2017), the measured DVC uncertainties had decreasing tends with respect to the sub-volume size for both types of specimens (Fig. 5). In particular, the median values for MAER and SDER for the bone-biomaterial samples ranged between $671 \mu \varepsilon$ to $167 \mu \varepsilon$ and $766 \mu \varepsilon$ to $42 \mu \varepsilon$ for the raw images, between $695 \mu \varepsilon$ to $154 \mu \varepsilon$ and $679 \mu \varepsilon$ to $44 \mu \varepsilon$ for the mask images, and between $1525 \mu \varepsilon$ to $208 \mu \varepsilon$ and $1354 \mu \varepsilon$ to $71 \mu \varepsilon$ for the DaVis-mask images, respectively; using subvolumes sizes ranging from 16 to 112 voxels. The errors for bone-biomaterial systems were lower than those obtained for the trabecular bone specimens. The median values of MAER and SDER for the bone-biomaterial samples ranged 
between $671 \mu \varepsilon$ to $167 \mu \varepsilon$ and $766 \mu \varepsilon$ to $42 \mu \varepsilon$ for the raw images, between $695 \mu \varepsilon$ to $154 \mu \varepsilon$ and $679 \mu \varepsilon$ to $44 \mu \varepsilon$ for the mask images, and between $1525 \mu \varepsilon$ to $208 \mu \varepsilon$ and $1354 \mu \varepsilon$ to $71 \mu \varepsilon$ for the DaVis-mask images, respectively; for the same subvolumes. As for the displacement random errors, the use of DaVis-mask considerably enlarged the measured strain uncertainties. The multi-pass scheme (final sub-volume size of 48 voxels) notably improved the performance in both types of specimens when compared to the results obtained with sub-volumes of 48 voxels for both MAER and SDER.

\section{Random errors for each strain component}

Increasing the sub-volume size reduced the random error of each strain component for both types of specimens and the different mask-based options used. As found for the displacement random errors, MAER and SDER, the bone-biomaterial systems were less affected when compared to the trabecular bone specimens. Consistently with previous results, the use of DaVis-mask images produced higher random errors for all strain components. Furthermore, the use of masked images and multi-pass scheme reduced the uncertainties for both types of specimens when compared to raw images and single-pass schemes (Fig. 6). For a final sub-volume of 48 voxels using a single-pass scheme, bone-biomaterials systems were associated to median random errors of 194 - $483 \mu \varepsilon$, and 161 - $544 \mu \varepsilon$ for raw and mask images, respectively. The use of a multi-pass scheme reduced the random errors to 171 $304 \mu \varepsilon$, and 112 - $338 \mu \varepsilon$ for raw and mask images, respectively. Similarly, for the trabecular bone specimens the random errors were found to be between 485 - 731 $\mu \varepsilon$ for the raw images, and 562 - $839 \mu \varepsilon$ for the mask images when using a singlepass scheme and between $261-436 \mu \varepsilon$ for raw images, and $205-426 \mu \varepsilon$ for mask images in the multi-pass scheme.

\section{Spatial distribution of the errors}

Generally, larger errors were found for the trabecular bone specimens compared to the bone-biomaterial systems. In particular, the distribution of the apparent normal strain in the z-direction (chosen as a representative strain component for the obtained results) seemed to be more homogeneous in the bone-biomaterial systems when compared to the trabecular bone (Fig. 7). As the DVC analysis is based on 'zero-strain' repeated scans, the strain distribution in Fig. 7 depicted the error distribution for the $\varepsilon_{z z}$ strain component. In areas presenting bone-biomaterial, a reasonably uniform distribution of the strain was obtained. Conversely, for the trabecular bone sample and areas of trabecular bone in the bone-biomaterial, larger strain errors and a more heterogeneous strain distribution was observed.

\section{DISCUSSION}

The goal of this study was to evaluate the influence of imaging post-processing and DVC settings on the displacement and strain error distribution within trabecular bone 
and bone-biomaterial systems, using a SR-microCT based local DVC approach. More specifically, this work aimed at optimising those settings in order to provide accurate 3D strain measurements, at tissue level, of bone-biomaterial interfaces for further micromechanical characterisation of such composites under applied load.

The application of DVC based on high-resolution SR-microCT images of bone remains partially unexplored. In fact, to the author's knowledge only two publications reported the used of DVC for 3D strain measurement on bone. Christen et al. (Christen et al., 2012) focused on crack propagation in murine femora, but the uncertainties of the measured strain were only assessed in virtually displaced images and not in repeated scans. Therefore, the real error induced by image noise was not taken into account, possibly leading to an underestimation of the errors (Dall'Ara et al., 2014). More recently, Palanca at al. (Palanca et al., 2017) showed that reliable strain measurements could be obtained at tissue-level using a global DVC approach for trabecular bone, cortical bone and murine tibia, but the performance of a local DVC approach and the study of bone-biomaterial interfaces was not explored. A comparison of two DVC algorithms (global and local approaches) in different bone typologies at different dimensional scales based on laboratory microCT and SR-microCT was conducted by Dall'Ara et al. (Dall'Ara et al., 2017), including the precision of a local approach of bone-biomaterial systems also investigated in this study. However, only the precision of the DVC algorithm in terms of strain and displacements was reported, and the uncertainties were evaluated on the entire 3D images, producing slightly higher errors when compared to an evaluation exclusively within the mineralised tissue. In fact, the present study showed that when computing the strain values only in the tissue, the SDER for bonebiomaterial systems was found to be $\sim 100 \mu \varepsilon$ whereas Dall'Ara reported $\sim 150 \mu \varepsilon$, for the same multi-pass scheme with a final sub-volume of 48 voxels. Furthermore, a comparison of the influence of different imaging post-processes and DVC settings on the resulting measurement uncertainties was not detailed.

Despite the high potential of using high-quality tomograms acquired with SRmicroCT on DVC applications of bone and bone-materials, concerns are still raising on the damage induced by SR X-ray radiation (Barth et al., 2010). In fact, when prolonged exposures times to SR X-ray radiation are required, the microstructural integrity of the bone tissue is compromised (Fig. 1), and microcracks appear clearly visible in the tissue after continuous irradiation. Therefore, this study used low exposures times (64 ms per projection, 2 min scanning time) and kept the specimens immersed in saline solution during image acquisition, in order preserve the mechanical integrity of the analysed specimens (Peña-Fernández et al., 2018). These two factors negatively contributed to the quality of the acquired tomograms (Fig. 2), and further optimisation on the imaging and DVC settings was needed.

In order to understand the effect of filtering the images prior to DVC computation, two different filters were applied and compared to the DVC results obtained with the 
raw noisy tomograms (Table 1). For instance, this is the first time that the performance of DVC is compared for the same images with different denoised methods, even though these are common practice in image post-processing prior to DVC computation (Palanca et al., 2017). It was shown that the use of advanced filters (i.e. NLM filter), not only improved the correlation coefficient but also slightly reduced the uncertainties for both displacement and strain measurements in both types of specimens, suggesting that image denoising should be carefully considered and evaluated when low-quality tomograms are acquired. Furthermore, the use of robust filters is an essential step before image segmentation (Fig. 3) prior to masking.

In this study an iterative approach for image segmentation was adopted, allowing the discrimination of mineralised tissue and biomaterial from soft/watery material. Despite segmenting images of bone acquired via microCT is commonly performed using global thresholds set manually, those introduce inter-observer variation (Waarsing et al., 2004). Local adaptive algorithms (Kaipala et al., 2017) can successfully segment images with strong edges (high contrast) and relatively uniform signal intensity. However, low-quality tomograms like those in the current study required a different segmentation approach. The iterative approach used in this study showed good visual agreement to the grayscale image (Fig. 3). However, the quality of the segmentation was only checked visually, resulting on an operatordependent approach; thus introducing inter-observer variation. Although stronger iterative thresholding algorithms (Wu et al., 2000) have proven to provide accurate results in terms of bone volume fraction, specific surface, and surface curvature (Slyfield et al., 2009), it was not within the scope of this paper to evaluate the morphology of the analysed specimen, but only the performance of DVC when artifacts presented in the non-bone are included. Therefore, a validation of the segmentation procedure herein applied was not conducted, and stronger methods were not tested.

The effect of the masking operation on the trabecular bone and bone-biomaterial composites was evaluated for the first time on a local DVC approach. Previously results on a global DVC approach based on masked images showed lower error compared to the ones obtained by raw images (Palanca et al., 2017), in agreement with the results of this study (Table 2). The exclusion of non-mineralised areas, for which noise and artifacts (i.e. bubbles in saline solution) were probably dominant, was beneficial for DVC registration when the 'background' was treated as 'zero' intensity, enhancing the correlation coefficient and lowering the errors. However, the use of a threshold-based algorithmic mask (DaVis-mask), in which the 'background' region is excluded from DVC computation, provided higher errors and lower correlation values. That approach to masking may work for masking away regions outside the analysed specimen (i.e. to mask away regions of tooth structure in boneperiodontal ligament and tooth fibrous joint (Jang et al., 2016)). However, when the masking is performed at tissue level in trabecular structure, DVC algorithm is not 
able to provide a good correlation if the edges between trabeculae and marrow (high intensity gradient) are excluded from the computation and only gradients within the trabecula are considered. In this sense, even a variation on the mfvp (Table 2) was not able to substantially improve the results, as that parameters affects only to the number of voxels to be contained within a sub-volume for being included in the computation (Fig. 4). Despite that, the computed SDER using DaVis-mask was found to be $\sim 400$ for the bone-biomaterial systems and $\sim 650$ for trabecular bone using a multi-pass approach with a final sub-volume of 48 voxels. Those values are still tolerable for investigating the deformation of both types of specimens at tissue level. Considering that the use of DaVis-mask is the only approach that accounts for the displacement/strain field only in the tissue the results seem promising, and further development of this approach should be conducted. In fact, the application of DaVis-mask to high resolution images of trabecular bone at higher signal-to-noise ratio may improve the performance of the algorithm, as more features would be seen within the trabeculae.

In line with previous studies (Dall'Ara et al., 2017; Palanca et al., 2017), the larger the sub-volume size, the lower the measurement uncertainties for both trabecular bone and bone-biomaterials (Fig. 5). Furthermore, it was reaffirmed (Palanca et al., 2015) that the multi-pass approach available in DaVis provided lower errors when compared to the same final sub-volume using a single-pass (Fig. 6). For a subvolume size of 48 voxels or larger, equivalent to $\sim 125 \mu \mathrm{m}$, the SDER was found close to $100 \mu \varepsilon$ for both trabecular bone and bone-biomaterial composites, whereas random error for each strain component was close to or below $300 \mu \varepsilon$ for the bonebiomaterial systems and close to or below $400 \mu \varepsilon$ for trabecular bone. These values are acceptable for the investigation of the deformation in the physiological range (1000-2000 $\mu \varepsilon$ (Yang et al., 2011)) at tissue level (Fratzl et al., 2004). Slightly higher errors were found for the trabecular bone compared to the results reported by Palanca et al. (Palanca et al., 2017). This was probably due to the differences in the effective pixel size (larger in this study) and the signal-to-noise ratio, lower in this work due to the low exposure times used to minimise the irradiation-induced damage in the tissue. Furthermore, the difference DVC approach (local vs global) may influence the measurement uncertainties based on the same datasets. In this sense, a comparison between global and local DVC approaches based on high-resolution SR-microCT images remains unexplored. However, the measurement uncertainties were lower compared to local DVC approach based on laboratory microCT systems (Dall'Ara et al., 2017). Better results were found for the bone-biomaterial systems compared to the trabecular bone, likely due to the much higher number of features present in such composites. Similar results were found in Tozzi et al. (2017), in which the presence of bone cement in vertebral bodies strongly modified the material texture, and therefore, positively influence the DVC analysis. In fact, the analysis of the spatial distribution of the errors (Fig. 7) confirmed that hypothesis: the areas with higher errors in the bone-biomaterials were correlated to native trabecular bone regions. 
The current study has some limitations. Firstly, only two trabecular bone controls and four bone-biomaterial systems were analysed; thus, no statistical information can be extracted. The size of the bone defects and the short time allocated in the beamline made not possible to enlarge the sample size. Additionally, the strain errors were only calculated in a 'zero-strain' condition for repeated scans. Despite this approach allows to account for the intrinsic image noise, this analysis should be expanded in order to evaluate the error within strained specimens. Particularly, Dall'Ara et al. (2017) suggested to overcome this limitation by evaluating the precision of the DVC approach on repeated scans of the structure under load. Despite they found that the precision was similar for both loaded and unloaded structures, the accuracy could not be evaluated as the displacement field is unknown. On the other hand, Palanca et al. (2017) proposed to perform the analysis in synthetically deformed images after imposing an affine transformation on the unloaded repeated scans, thus not accounting for a realistic heterogeneous strain field. Future work must be done to account for more realistic loading scenarios.

In conclusion, this study demonstrated the suitability of a local DVC approach based on SR-microCT images to investigate the micromechanics of trabecular bone and bone-biomaterial systems at tissue level. This was achieved after an optimisation of image post-processing and DVC settings. Image quality had to be reduced by decreasing the exposure time to $S R X$-ray radiation to minimise irradiation-induce tissue damage. The use of advanced 3D filters on the acquired dataset enhanced DVC computation and provided a better segmentation of bone and biomaterial. The computation of displacement and strain values only in the mineralised tissue and biomaterial allowed for the exclusion of artifacts, resulting in lower errors. This approach has proven to be valid to evaluate full-field strain in bone-biomaterial composites under load at the tissue level $(\sim 150 \mu \mathrm{m})$, with a standard deviation of the errors of $\sim 100 \mu \varepsilon$.

\section{ACKNOWLEDGMENTS}

The authors would like to thank Diamond Light Source for time at the DiamondManchester Imaging Branchline I13-2 and I13 Data Beamline (Bodey \& Rau, 2017) (proposal number MT14080), and the Zeiss Global Centre (University of Portsmouth) for fast-processing. We further acknowledge Dr. David Hollis and Dr. Manuel Grewer (LaVision Ltd) for assistance with DaVis software and Dr. Andrew Bodey, Dr. Krazimir Wanelik and Rachna Parwani for help with the acquisition of the images at Diamond Light Source. The project was partially funded by Innovate UK Innovation Vouchers and LaVision Ltd UK.

\section{REFERENCES}

Atwood, R. C., Bodey, A. J., Price, S. W. T., Basham, M., \& Drakopoulos, M. (2015). A high-throughput system for high-quality tomographic reconstruction of large datasets at Diamond Light Source. Philosophical Transactions of the Royal 
Society A: Mathematical, Physical and Engineering Sciences, 373(2043). https://doi.org/10.1098/rsta.2014.0398

Barth, H. D., Launey, M. E., MacDowell, A. A., Ager, J. W., \& Ritchie, R. O. (2010). On the effect of X-ray irradiation on the deformation and fracture behavior of human cortical bone. Bone, 46(6), 1475-1485. https://doi.org/10.1016/j.bone.2010.02.025

Basham, M., Filik, J., Wharmby, M. T., Chang, P. C. Y., El Kassaby, B., Gerring, M., ... Ashton, A. W. (2015). Data Analysis WorkbeNch (DAWN). Journal of Synchrotron Radiation, 22, 853-858. https://doi.org/10.1107/S1600577515002283

Bay, B. K., Smith, T. S., Fyhrie, D. P., \& Saad, M. (1999). Digital volume correlation: Three-dimensional strain mapping using X-ray tomography. Experimental Mechanics, 39(3), 217-226. https://doi.org/10.1007/BF02323555

Bodey, A. J., \& Rau, C. (2017). Launch of the I13-2 data beamline at the Diamond Light Source synchrotron. Journal of Physics: Conference Series, 849(1). https://doi.org/10.1088/1742-6596/849/1/012038

Buades, A., Coll, B., \& Morel, J.-M. (2011). Non-Local Means Denoising. Image Processing On Line, 1, 490-530. https://doi.org/10.5201/ipol.2011.bcm_nlm

Buffiere, J. Y., Maire, E., Adrien, J., Masse, J. P., \& Boller, E. (2010). In situ experiments with $X$ ray tomography: An attractive tool for experimental mechanics. Proceedings of the Society for Experimental Mechanics, Inc., 67, 289-305. https://doi.org/10.1007/s11340-010-9333-7

Cheminet, A., Leclaire, B., Champagnat, F., Plyer, A., Yegavian, R., \& Besnerais, G. Le. (2014). Accuracy assessment of a Lucas-Kanade based correlation method for 3D PIV. 17th International Symposium on Applications of Laser Techniques to Fluid Mechanics, 7-10.

Christen, D., Levchuk, A., Schori, S., Schneider, P., Boyd, S. K., \& Müller, R. (2012). Deformable image registration and $3 \mathrm{D}$ strain mapping for the quantitative assessment of cortical bone microdamage. Journal of the Mechanical Behavior of Biomedical Materials, 8, 184-193. https://doi.org/10.1016/j.jmbbm.2011.12.009

Coathup, M. J., Edwards, T. C., Samizadeh, S., Lo, W. J., \& Blunn, G. W. (2016). The effect of an alginate carrier on bone formation in a hydroxyapatite scaffold. Journal of Biomedical Materials Research - Part B Applied Biomaterials, 104(7), 1328-1335. https://doi.org/10.1002/jbm.b.33395

Dall'Ara, E., Barber, D., \& Viceconti, M. (2014). About the inevitable compromise between spatial resolution and accuracy of strain measurement for bone tissue: A 3D zero-strain study. Journal of Biomechanics, 47(12), 2956-2963. https://doi.org/10.1016/j.jbiomech.2014.07.019

Dall'Ara, E., Peña-Fernández, M., Palanca, M., Giorgi, M., Cristofolini, L., \& Tozzi, G. (2017). Precision of DVC approaches for strain analysis in bone imaged with $\mu C T$ at different dimensional levels. Frontiers in Materials, 4:31. https://doi.org/10.3389/fmats.2017.00031

Danesi, V., Tozzi, G., \& Cristofolini, L. (2016). Application of digital volume correlation to study the efficacy of prophylactic vertebral augmentation. Clinical Biomechanics, 39, 14-24. https://doi.org/10.1016/j.clinbiomech.2016.07.010

Darbon, J., Cunha, A., Chan, T. F., Osher, S., \& Jensen, G. J. (2008). Fast nonlocal filtering applied to electron cryomicroscopy. In 2008 5th IEEE International Symposium on Biomedical Imaging: From Nano to Macro, Proceedings, ISBI. IEEE, 1331-1334. https://doi.org/10.1109/ISBI.2008.4541250 
Dorozhkin, S. V. (2013). Calcium Orthophosphate-Based Bioceramics. Materials, 6, 3840-3942. https://doi.org/10.3390/ma6093840

Doube, M., Klosowski, M. M., Arganda-Carreras, I., Cordelières, F. P., Dougherty, R. P., Jackson, J. S., ... Shefelbine, S. J. (2010). BoneJ: Free and extensible bone image analysis in ImageJ. Bone, 47(6), 1076-1079. https://doi.org/10.1016/j.bone.2010.08.023

Fratzl, P., Gupta, H. S., Paschalis, E. P., \& Roschger, P. (2004). Structure and mechanical quality of the collagen-mineral nano-composite in bone. J. Mater. Chem., 14(14), 2115-2123. https://doi.org/10.1039/B402005G

García-Gareta, E., Coathup, M. J., \& Blunn, G. W. (2015). Osteoinduction of bone grafting materials for bone repair and regeneration. Bone, 81, 112-121. https://doi.org/10.1016/j.bone.2015.07.007

Germaneau, A., Doumalin, P., \& Dupré, J. C. (2007a). 3D strain field measurement by correlation of volume images using scattered light: Recording of images and choice of marks. Strain, 43(3), 207-218. https://doi.org/10.1111/j.14751305.2007.00340.x

Germaneau, A., Doumalin, P., \& Dupré, J. C. (2007b). Full 3D Measurement of Strain Field by Scattered Light for Analysis of Structures. Experimental Mechanics, 47(4), 523-532. https://doi.org/10.1007/s11340-006-9029-1

Gillard, F., Boardman, R., Mavrogordato, M., Hollis, D., Sinclair, I., Pierron, F., \& Browne, M. (2014). The application of digital volume correlation (DVC) to study the microstructural behaviour of trabecular bone during compression. Journal of the Mechanical Behavior of Biomedical Materials. https://doi.org/10.1016/j.jmbbm.2013.09.014

Grassi, L., \& Isaksson, H. (2015). Extracting accurate strain measurements in bone mechanics: A critical review of current methods. Journal of the Mechanical Behavior of Biomedical Materials, 50, 43-54. https://doi.org/10.1016/j.jmbbm.2015.06.006

Huang, L.-K., \& Wang, M.-J. J. (1955). Image thresholding by minimizing the measures of fuzziness. Pattern Recognition, 28(1), 41-51.

Hussein, A. I., Barbone, P. E., \& Morgan, E. F. (2012). Digital volume correlation for study of the mechanics of whole bones. Procedia IUTAM, 4, 116-125. https://doi.org/10.1016/j.piutam.2012.05.013

Immerkær, J., (1996). Fast noise variance estimation. Computer Vision and Image Understanding, 64(2), pp.300-302.

Jang, A., Prevost, R., \& Ho, S. P. (2016). Strain mapping and correlative microscopy of the alveolar bone in a bone-periodontal ligament-tooth fibrous joint. Proceedings of the Institution of Mechanical Engineers, Part $\mathrm{H}$ : Journal of Engineering in Medicine, 230(9), 847-857. https://doi.org/10.1177/0954411916655183

Kaipala, J., Bordallo, M., \& Saarakkala, S. (2017). Automatic Segmentation of Bone Tissue from Computed Tomography Using a Volumetric Local Binary Patterns Based Method. Scandinavian Conference on Image Analysis, 221-232. https://doi.org/10.1007/978-3-319-59129-2

Keaveny, T. M., Borchers, R. E., Gibson, L. J., \& Hayes, W. C. (1993). Theoretical analysis of the experimental artifact in trabecular bone compressive modulus. Journal of Biomechanics, 25(4/5), 599-607.

Liu, L., \& Morgan, E. F. (2007). Accuracy and precision of digital volume correlation in quantifying displacements and strains in trabecular bone. Journal of Biomechanics, 40(15), 3516-3520. 
https://doi.org/10.1016/j.jbiomech.2007.04.019

Madi, K., Tozzi, G., Zhang, Q. H., Tong, J., Cossey, A., Au, A., ... Hild, F. (2013a). Computation of full-field displacements in a scaffold implant using digital volume correlation and finite element analysis. Medical Engineering and Physics. https://doi.org/10.1016/j.medengphy.2013.02.001

Madi, K., Tozzi, G., Zhang, Q. H., Tong, J., Cossey, A., Au, A., ... Hild, F. (2013b). Computation of full-field displacements in a scaffold implant using digital volume correlation and finite element analysis. Medical Engineering and Physics, 35(9), 1298-1312. https://doi.org/10.1016/j.medengphy.2013.02.001

Meijering, E. H. W., Niessen, W. J., \& Viergever, M. A. (2001). Quantitative Evaluation of Convolution-Based Methods for Medical Image Interpolation. Medical Image Analysis, 5(2), 111-126. https://doi.org/doi.org/10.1016/S13618415(00)00040-2

Nazarian, A., \& Müller, R. (2004). Time-lapsed microstructural imaging of bone failure behavior. Journal of Biomechanics, 37(1), 55-65. https://doi.org/10.1016/S0021-9290(03)00254-9

Odgaard, A., \& Gundersen, H. J. G. (1993). Quantification of connectivity in cancellous bone, with special emphasis on 3-D reconstructions. Bone, 14(2), 173-182. https://doi.org/https://doi.org/10.1016/8756-3282(93)90245-6

Palanca, M., Bodey, A. J., Giorgi, M., Viceconti, M., Lacroix, D., Cristofolini, L., \& Dall'Ara, E. (2017). Local displacement and strain uncertainties in different bone types by digital volume correlation of synchrotron microtomograms. Journal of Biomechanics, c. https://doi.org/10.1016/j.jbiomech.2017.04.007

Palanca, M., Cristofolini, L., Dall'Ara, E., Curto, M., Innocente, F., Danesi, V., \& Tozzi, G. (2016). Digital volume correlation can be used to estimate local strains in natural and augmented vertebrae: an organ-level study. Journal of Biomechanics, 49(16), 3882-3890. https://doi.org/10.1016/j.jbiomech.2016.10.018

Palanca, M., Tozzi, G., \& Cristofolini, L. (2015). The use of digital image correlation in the biomechanical area: a review. Internatlonal Blomechanlcs, 3(1), 1-21. https://doi.org/10.1080/23335432.2015.1117395

Palanca, M., Tozzi, G., Cristofolini, L., Viceconti, M., \& Dall'Ara, E. (2015). 3D Local Measurements of Bone Strain and Displacement: Comparison of Three Digital Volume Correlation Approaches. Journal of Biomechanical Engineering, 137(July), 1-14. https://doi.org/10.1115/1.4030174

Peña-Fernández, M., Cipiccia, S., Bodey, A. J., Parwani, R., Dall'Ara, E., Blunn, G., ... Tozzi, G. (2018). Effect of SR-microCT exposure time on the mechanical integrity of trabecular bone using in situ mechanical testing and digital volume correlation (Under Review)

Roberts, B. C., Perilli, E., \& Reynolds, K. J. (2014). Application of the digital volume correlation technique for the measurement of displacement and strain fields in bone: A literature review. Journal of Biomechanics. https://doi.org/10.1016/j.jbiomech.2014.01.001

Scarano, F. (2013). Tomographic PIV: Principles and practice. Measurement Science and Technology, 24(1). https://doi.org/10.1088/0957-0233/24/1/012001

Schindelin, J., Arganda-Carreras, I., Frise, E., Kaynig, V., Longair, M., Pietzsch, T., ... Cardona, A. (2012). Fiji: an open-source platform for biological-image analysis. Nature Methods, 9(7), 676-682. https://doi.org/10.1038/nmeth.2019

Slyfield, C. R., Niemeyer, K. E., Tkachenko, E. V., Tomlinson, R. E., Steyer, G. G., Patthanacharoenphon, C. G., ... Hernandez, C. J. (2009). Three-Dimensional 
Surface Texture Visualization of Bone Tissue Through Epifluorescence-Based Serial Block Face Imaging. Journal of Microscopy, 236(1), 52-59. https://doi.org/10.1037/a0015862.Trajectories

Sriranganathan, D., Kanwal, N., Hing, K. A., \& Hill, R. G. (2016). Strontium substituted bioactive glasses for tissue engineered scaffolds: the importance of octacalcium phosphate. Journal of Materials Science: Materials in Medicine, 27(2), 1-10. https://doi.org/10.1007/s10856-015-5653-6

Stevens, M. M. (2008). Biomaterials for bone tissue engineering. Materials Today, 11(5), 18-25. https://doi.org/10.1016/S1369-7021(08)70086-5

Titarenko, V., Bradley, R., Martin, C., Withers, P. J., \& Titarenko, S. (2010). Regularization methods for inverse problems in x-ray tomography. Proc. SPIE 7804, (Developments in X-Ray Tomography VII). https://doi.org/doi: 10.1117/12.860260

Tozzi, G., Dall, E., Palanca, M., Curto, M., Innocente, F., \& Cristofolini, L. (2017). Journal of the Mechanical Behavior of Biomedical Materials Strain uncertainties from two digital volume correlation approaches in prophylactically augmented vertebrae : Local analysis on bone and cement- bone microstructures. Journal of the Mechanical Behavior of Biomedical Materials, 67(February 2016), 117-126. https://doi.org/10.1016/j.jmbbm.2016.12.006

Tozzi, G., Danesi, V., Palanca, M., \& Cristofolini, L. (2016). Elastic Full-Field Strain Analysis and Microdamage Progression in the Vertebral Body from Digital Volume Correlation. Strain, 52(5), 446-455. https://doi.org/10.1111/str.12202

Tozzi, G., De Mori, A., Oliveira, A., \& Roldo, M. (2016). Composite hydrogels for bone regeneration. Materials, 9(4), 1-24. https://doi.org/10.3390/ma9040267

Tozzi, G., Zhang, Q. H., \& Tong, J. (2012). 3D real-time micromechanical compressive behaviour of bone-cement interface: Experimental and finite element studies. Journal of Biomechanics. https://doi.org/10.1016/j.jbiomech.2011.10.011

Tozzi, G., Zhang, Q. H., \& Tong, J. (2014). Microdamage assessment of bonecement interfaces under monotonic and cyclic compression. Journal of Biomechanics. https://doi.org/10.1016/j.jbiomech.2014.09.012

Waarsing, J. H., Day, J. S., \& Weinans, H. (2004). An Improved Segmentation Method for In Vivo HCT Imaging. Journal of Bone and Mineral Research, 19(10), 1640-1650. https://doi.org/10.1359/JBMR.040705

Wang, W., \& Yeung, K. W. K. (2017). Bone grafts and biomaterials substitutes for bone defect repair: A review. Bioactive Materials, 2(4), 224-247. https://doi.org/10.1016/j.bioactmat.2017.05.007

Wu, H. S., Barba, J., \& Gil, J. (2000). Iterative thresholding for segmentation of cells from noisy images. Journal of Microscopy, 197(3), 296-304. https://doi.org/10.1046/j.1365-2818.2000.00653.x

Yang, P. F., Brüggemann, G. P., \& Rittweger, J. (2011). What do we currently know from in vivo bone strain measurements in humans? Journal of Musculoskeletal Neuronal Interactions, 11(1), 8-20. 


\section{FIGURE CAPTIONS}

Figure 1. SR-microCT two-dimensional (2D) cross-sections acquired at different exposure times in the same bone-biomaterial system (ApaPore). Increasing the exposure time per projection (from a to f) improved the quality of the images but induced damage in the tissue due to SR X-ray radiation. Red arrows indicate microcracks in the tissue. (a) $t_{\exp }=32 \mathrm{~ms}$. (b) $t_{\text {exp }}=64 \mathrm{~ms}$. (c) $t_{\exp }=128 \mathrm{~ms}$. (d) $t_{\exp }$ $=256 \mathrm{~ms}$. (e) $\mathrm{t}_{\mathrm{exp}}=512 \mathrm{~ms}$. (f) $\mathrm{t}_{\mathrm{exp}}=1024 \mathrm{~ms}$. (texp: exposure time per projection).

Figure 2. SR-microCT two-dimensional (2D) cross-sections in trabecular bone (a) and bone-biomaterial interface of Actifuse (b). The raw images (first column) present a reduced quality due to the low X-ray exposure used. Applying a median filter (second column) and a non-local means filter (third column) resulted in considerable noise reduction.

Figure 3. Iterative approach for segmentation of SR-microCT images of bonebiomaterial systems. (a) Non-local means denoised image showing unabsorbed biomaterial (light grey), bone tissue (medium grey) and non-mineralized material (dark grey). (b) Final mask image where non-mineralized voxels were set to zero (black) intensity value. (c) Initial segmentation based on Huang's method. Binary images after two (d), four (e) and six (f) iterations. More bone-biomaterial and less marrow/watery material is included in the segmentation as the number of iterations increases.

Figure 4. Normalized correlation coefficient $\left(\mathbf{C}_{\text {norm }}\right)$ overlaid to the 2D SR-microCT tomograms showing the influence of varying the minimal fraction of valid pixel ( $m f v p$ ) from $30 \%$ to $50 \%$ on the correlated volume for a trabecular bone specimen (a) and a bone-biomaterial interface (b). Areas with a high density of material (top area in $\mathbf{b}$ ) present a higher correlation compared to areas of trabecular bone (bottom area in b).

Figure 5. MAER (top) and SDER (bottom) for bone-biomaterial systems (left) and trabecular bone (right), for raw, mask and DaVis-mask images (blue, orange and green bars, respectively) as a function of the sub-volume size. Results for the multipass (mp) scheme are also shown. Bars represent the median value, while error bars represent the standard deviation between the number of specimens of each type.

Figure 6. Random errors of each strain component for bone-biomaterial systems (left) and trabecular bone (right), computed using raw (top) and mask (bottom) images for a final sub-volume size of 48 voxels using a single-pass (blue) and a multi-pass (yellow) scheme. Bars represent the median value, while error bars represent the standard deviation accounting for the total number of specimens in each type. 
Figure 7. Distribution of the z-direction strain $\left(\varepsilon_{z z}\right)$ component for a cross-section of a trabecular bone (a) and a bone-biomaterial specimen(c) and for their VOls (trabecular bone (b) and bone-biomaterial (d)), computed using a multi-pass scheme (48 voxel final sub-volume size) on the mask images. As the DVC was applied to 'zero-strain' repeated scans, the reported strain represents the measured DVC uncertainties 


\section{LAY DESCRIPTION}

Understanding the quality of newly regenerated bone after implantation of novel biomaterials is essential to improve bone tissue engineering strategies and formulation of biomaterials. The relationship between microstructure and mechanics of bone has been previously addressed combining micro-computed tomography (microCT) with in situ mechanical testing. The addition of an image-based experimental technique such as digital volume correlation (DVC) allows to characterise the deformation of materials in a three-dimensional manner. However, in order to obtain accurate information at the micro-scale, high-resolution images, obtained for example by using synchrotron radiation microCT (SR-microCT), as well as optimisation of the DVC computation are needed. This study presents the effect of different imaging parameters, image post-processing and DVC settings for as accurate investigation of trabecular bone structure and bone-biomaterial interfaces. The results showed that when appropriate image post-processing and DVC settings are used DVC computation results in very low strain errors. This is of vital importance for a correct understanding of the deformation in bone-biomaterial systems and the ability of such biomaterials in producing new bone comparable with the native tissue they are meant to replace. 

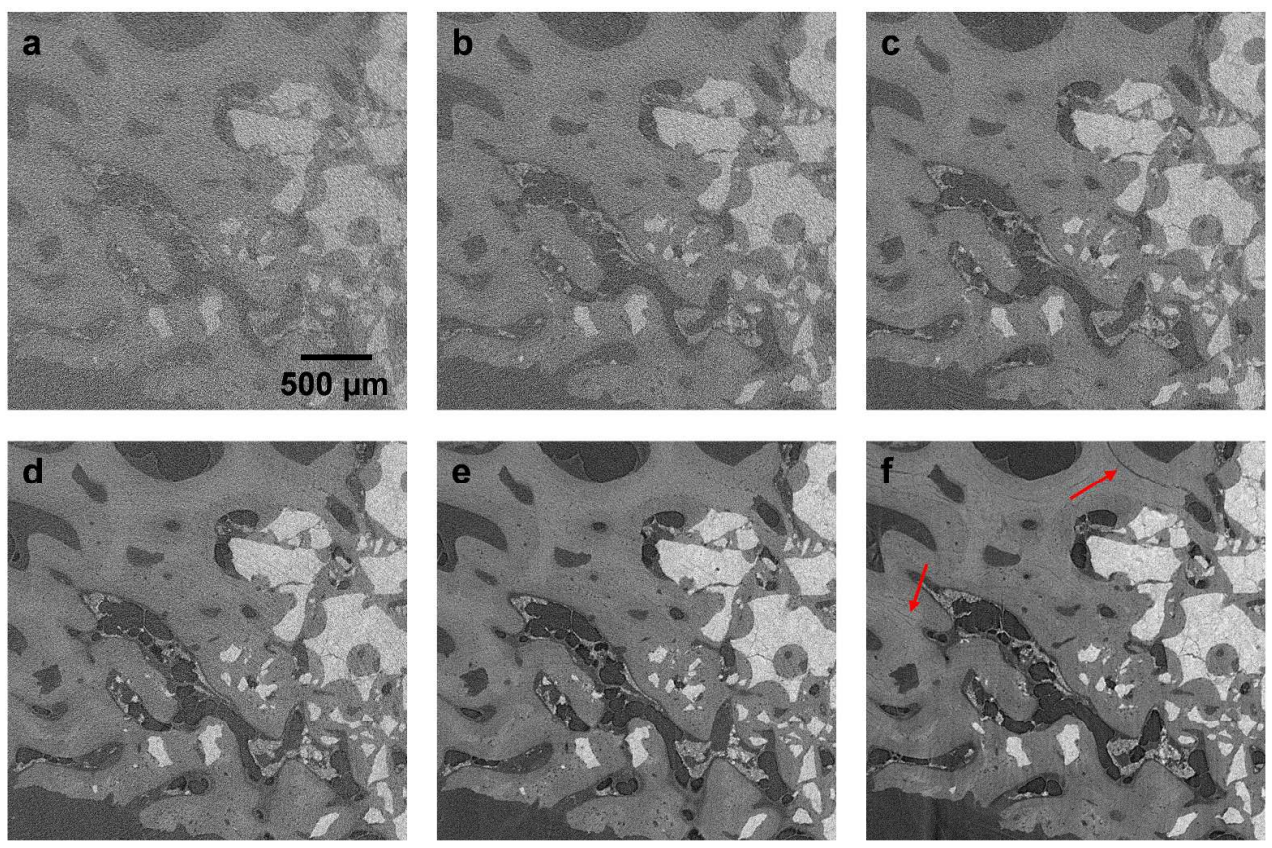

Figure 1. SR-microCT two-dimensional (2D) cross-sections acquired at different exposure times in the same bone-biomaterial system (ApaPore). Increasing the exposure time per projection (from a to f) improved the quality of the images but induced damage in the tissue due to SR X-ray radiation. Red arrows indicate microcracks in the tissue. (a) texp $=32 \mathrm{~ms}$. (b) texp $=64 \mathrm{~ms}$. (c) texp $=128 \mathrm{~ms}$. (d) texp $=256 \mathrm{~ms}$. (e) texp $=512 \mathrm{~ms}$. (f) texp $=1024 \mathrm{~ms}$. (texp: exposure time per projection). 
a
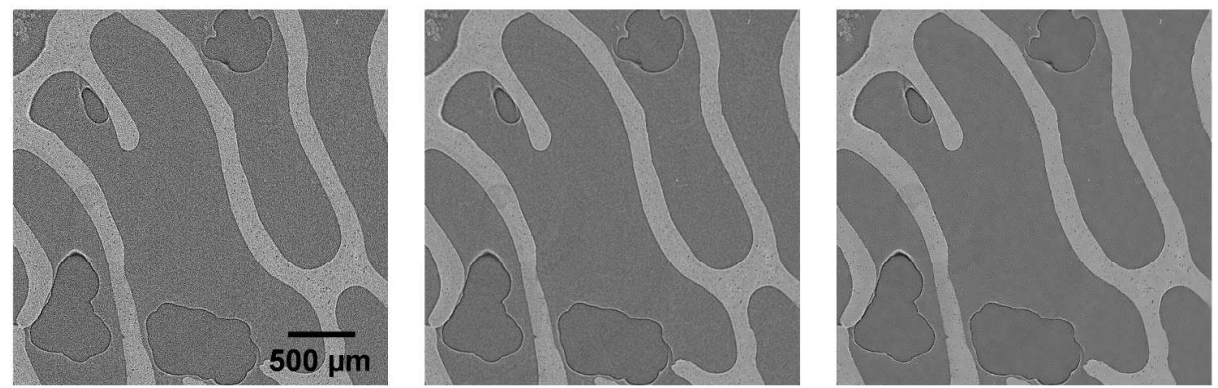

b

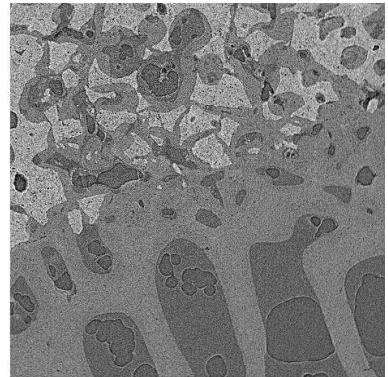

Raw images

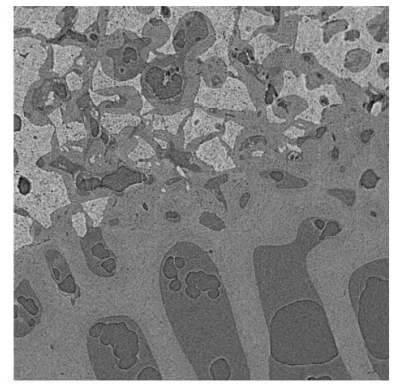

Median filtered images

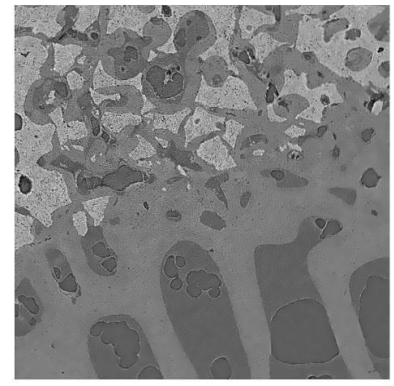

Non-local means denoised images

Figure 2. SR-microCT two-dimensional (2D) cross-sections in trabecular bone (a) and bone-biomaterial interface of Actifuse (b). The raw images (first column) present a reduced quality due to the low X-ray exposure used. Applying a median filter (second column) and a non-local means filter (third column) resulted in considerable noise reduction.

$473 \times 335 \mathrm{~mm}(300 \times 300$ DPI $)$ 

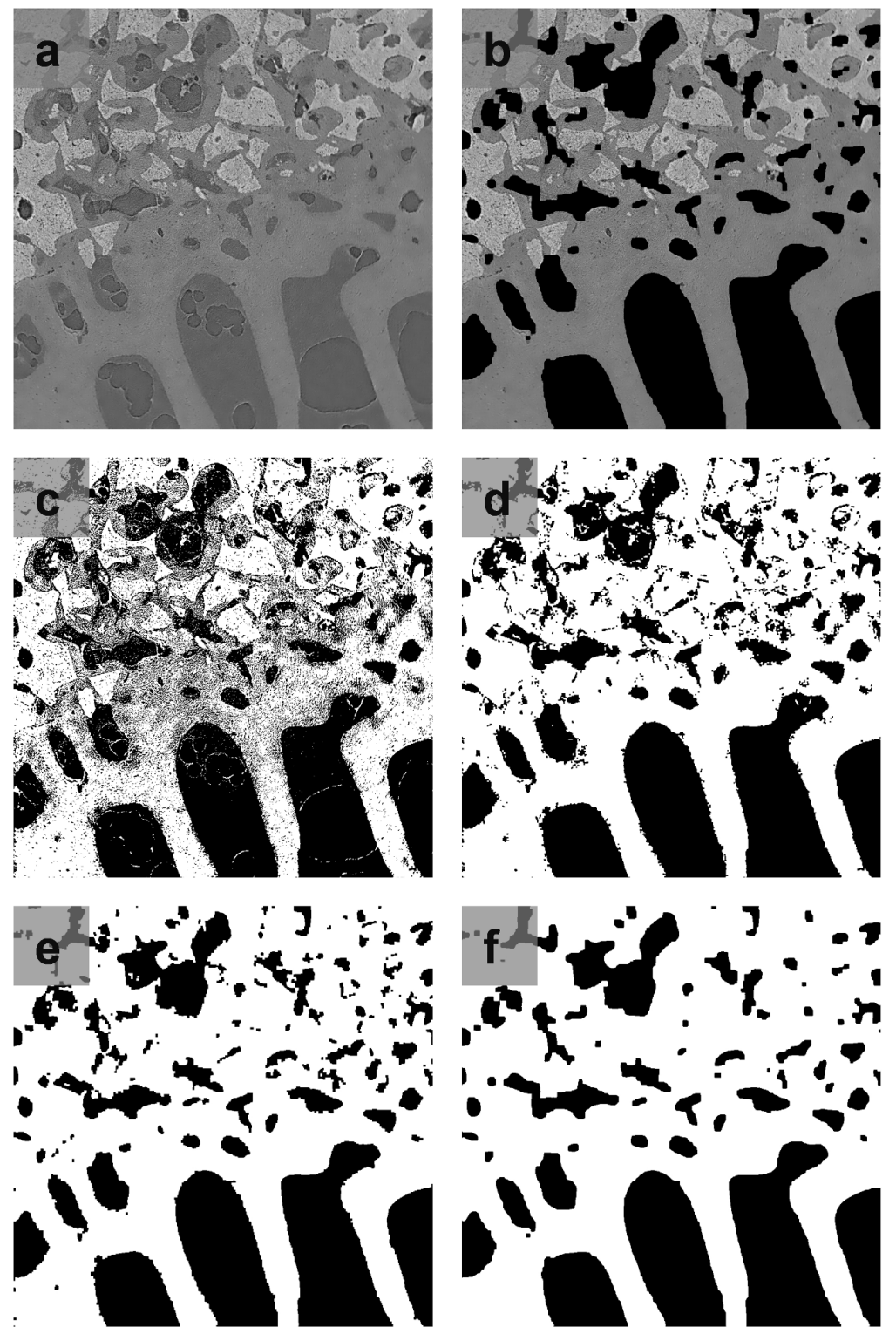

Figure 3. Iterative approach for segmentation of SR-microCT images of bone-biomaterial systems. (a) Nonlocal means denoised image showing unabsorbed biomaterial (light grey), bone tissue (medium grey) and non-mineralized material (dark grey). (b) Final mask image where non-mineralized voxels were set to zero (black) intensity value. (c) Initial segmentation based on Huang's method. Binary images after two (d), four

(e) and six (f) iterations. More bone-biomaterial and less marrow/watery material is included in the segmentation as the number of iterations increases. 
a
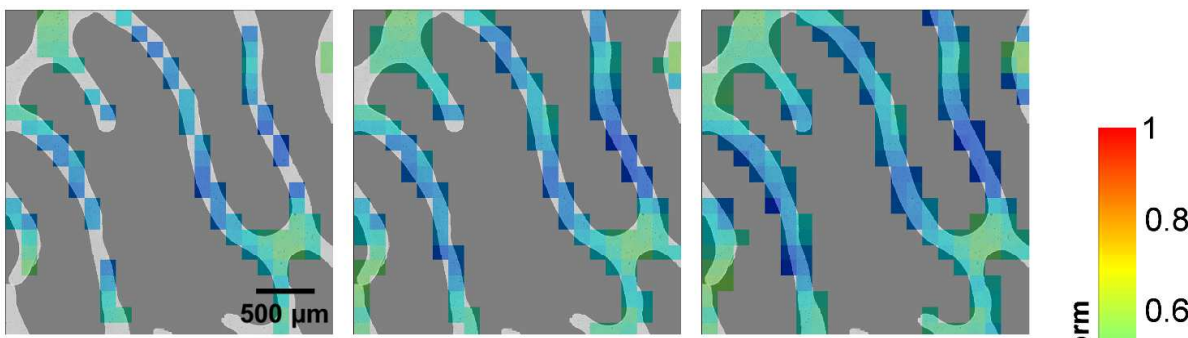

b

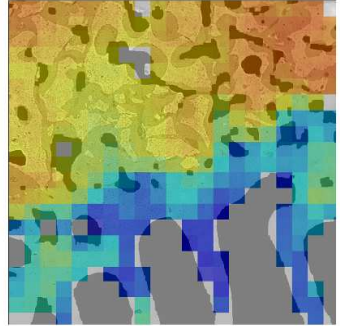

$50 \%$

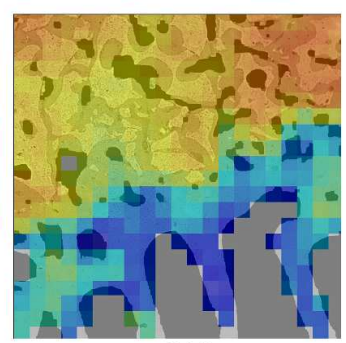

$40 \%$

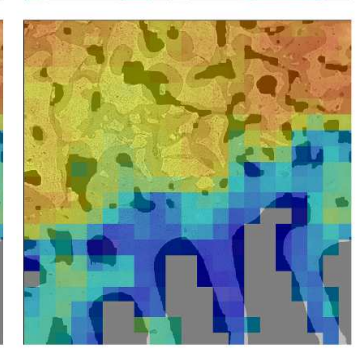

$30 \%$

Figure 4. Normalized correlation coefficient (Cnorm) overlaid to the 2D SR-microCT tomograms showing the influence of varying the minimal fraction of valid pixel ( $\mathrm{mfvp}$ ) from $30 \%$ to $50 \%$ on the correlated volume for a trabecular bone specimen (a) and a bone-biomaterial interface (b). Areas with a high density of material (top area in b) present a higher correlation compared to areas of trabecular bone (bottom area in b). 

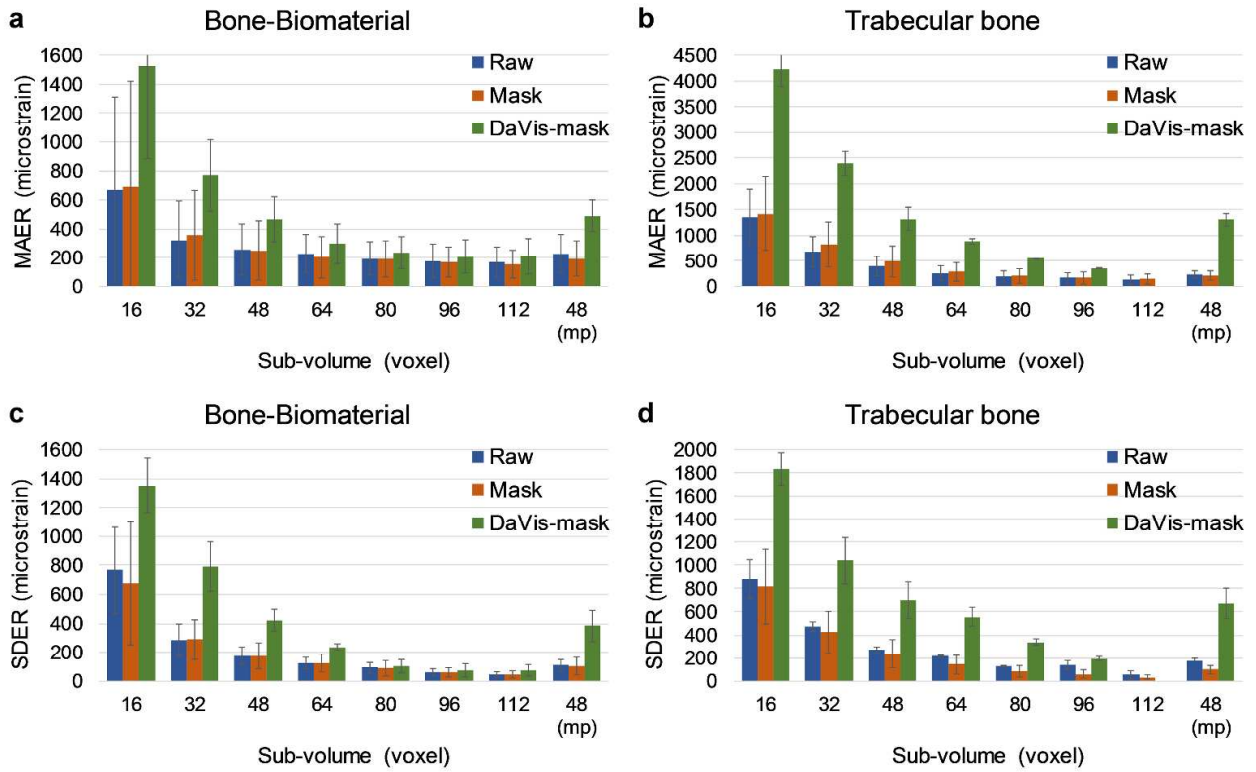

Figure 5. MAER (top) and SDER (bottom) for bone-biomaterial systems (left) and trabecular bone (right), for raw, mask and DaVis-mask images (blue, orange and green bars, respectively) as a function of the subvolume size. Results for the multi-pass $(\mathrm{mp})$ scheme are also shown. Bars represent the median value, while error bars represent the standard deviation between the number of specimens of each type. 

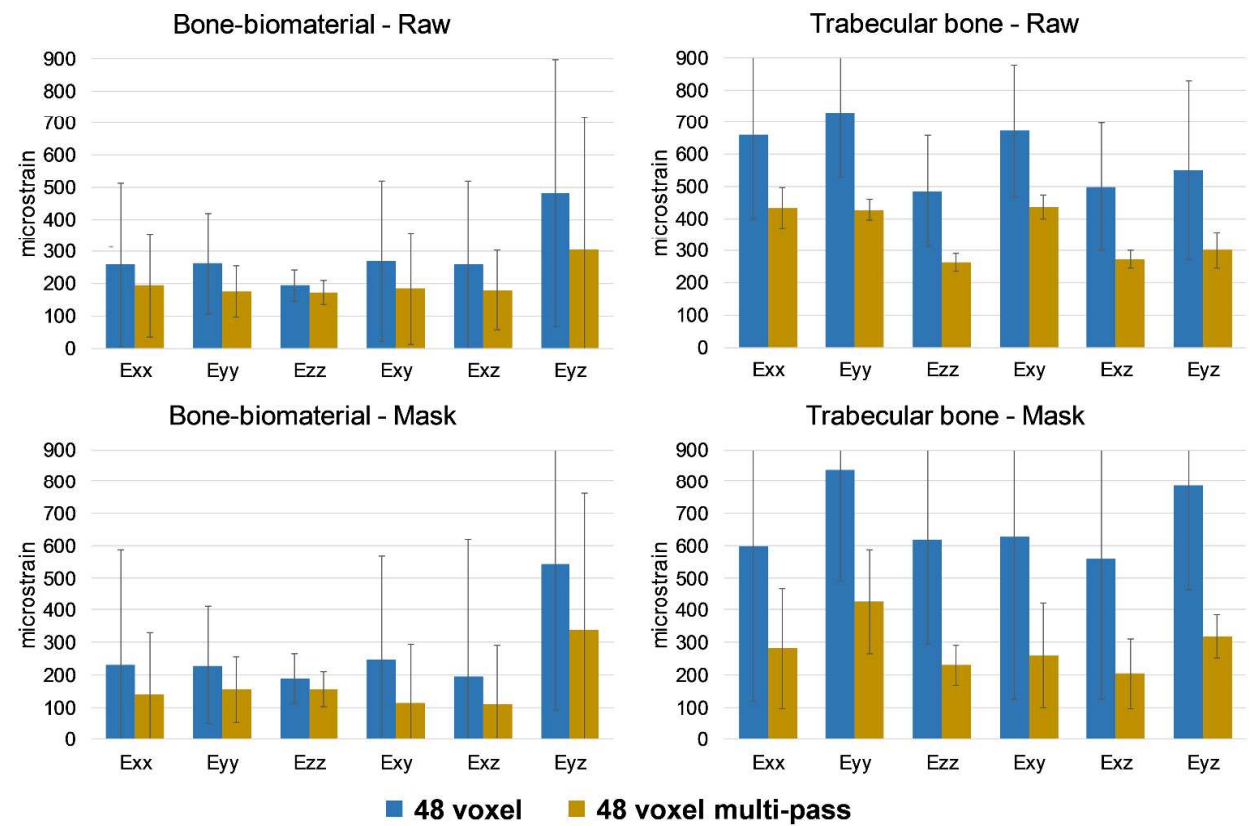

Figure 6. Random errors of each strain component for bone-biomaterial systems (left) and trabecular bone (right), computed using raw (top) and mask (bottom) images for a final sub-volume size of 48 voxels using a single-pass (blue) and a multi-pass (yellow) scheme. Bars represent the median value, while error bars represent the standard deviation accounting for the total number of specimens in each type. 

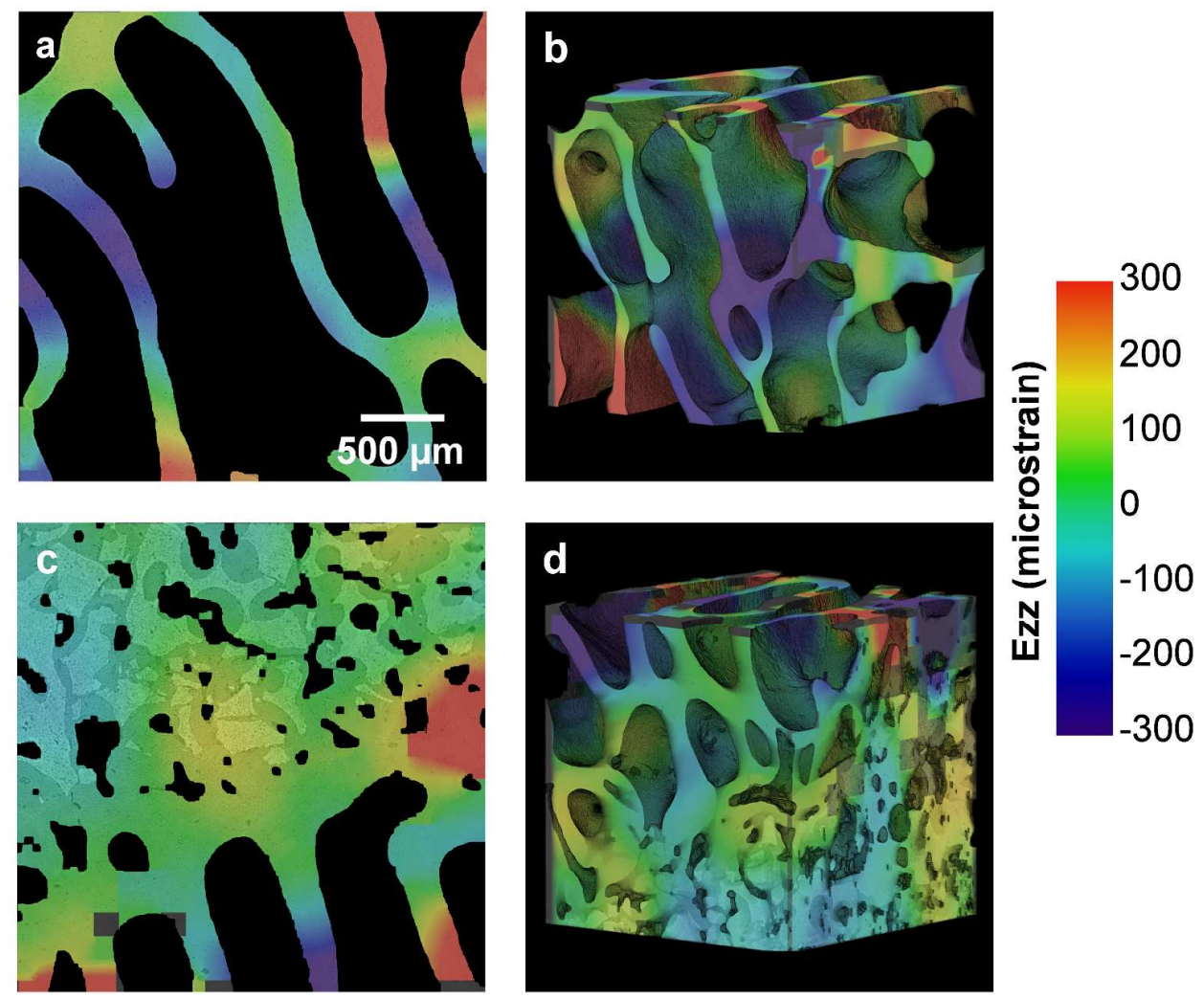

Figure 7. Distribution of the z-direction strain ( $\varepsilon z z)$ component for a cross-section of a trabecular bone (a) and a bone-biomaterial specimen(c) and for their VOIs (trabecular bone (b) and bone-biomaterial (d)), computed using a multi-pass scheme (48 voxel final sub-volume size) on the mask images. As the DVC was applied to 'zero-strain' repeated scans, the reported strain represents the measured DVC uncertainties 